\title{
Surface-tethering of methylated polyrotaxanes with 4-vinylbenzyl groups onto poly(ether ether ketone) substrates for improving osteoblast compatibility
}

\author{
Yoshinori ARISAKA ${ }^{1}$, Masahiro HAKARIYA², Takanori IWATA², Hiroki MASUDA ${ }^{3}$, Tetsuya YODA ${ }^{3}$, Atsushi TAMURA ${ }^{1}$ \\ and Nobuhiko YUI $^{1}$ \\ ${ }^{1}$ Department of Organic Biomaterials, Institute of Biomaterials and Bioengineering, Tokyo Medical and Dental University (TMDU), 2-3-10 Kanda- \\ Surugadai, Chiyoda-ku, Tokyo 101-0062, Japan \\ ${ }^{2}$ Department of Periodontology, Graduate School of Medical and Dental Sciences, Tokyo Medical and Dental University (TMDU), 1-5-45 Yushima, \\ Bunkyo-ku, Tokyo 113-8549, Japan \\ ${ }^{3}$ Department of Maxillofacial Surgery, Graduate School of Medical and Dental Sciences, Tokyo Medical and Dental University (TMDU), 1-5-45 \\ Yushima, Bunkyo-ku, Tokyo 113-8549, Japan \\ Corresponding author, Nobuhiko YUl; E-mail: yui.org@tmd.ac.jp
}

\begin{abstract}
Poly(ether ether ketone) (PEEK) is a high-performance thermoplastic used for several industrial applications due to its excellent mechanical properties. However, the use of PEEK is limited to dental materials because of its poor implant-bone integration. In the present study, methylated polyrotaxanes (MePRXs) with 4-vinylbenzyl groups, which are supermolecules composed of methylated $\alpha$-cyclodextrins and poly(ethylene glycol) chains end-capped with 4-vinylbenzyl groups, were covalently tethered onto PEEK surfaces using photo-induced polymerization to improve their osteoblast compatibility. The surface-tethering of MePRXs onto PEEK surfaces was confirmed by analyzing their attenuated total reflectance Fourier transform infrared spectra and contact angles. When mouse preosteoblasts were cultured on the MePRX-PEEK and bare PEEK surfaces, the MePRX-PEEK surfaces showed significantly better proliferation and osteoblast differentiation than the bare PEEK surfaces. These results suggest that surface modification of PEEKs using MePRXs improves their osteoblast compatibility.
\end{abstract}

Keywords: Polyrotaxane, Poly(ether ether ketone), Osteoblast differentiation

\section{INTRODUCTION}

Poly(oxy-1,4-phenyleneoxy-1,4-phenylenecarbonyl1,4-phenylene), commonly referred to as poly(ether ether ketone) (PEEK), is an engineering thermoplastic invented by Imperial Chemical Industries in 1977-3). Due to its low weight and mechanical robustness, PEEK has been used as a replacement for metals in various industrial applications, such as pumps, gears, insulators, and valves. In addition, PEEK has been investigated in the field of dentistry because it exhibits bone-like mechanical properties ${ }^{4}$, radiolucency, biocompatibility, nontoxicity, and clinically low inflammation ${ }^{5)}$. For instance, PEEK-based implants and maxillofacial prostheses have been developed ${ }^{6,7)}$, and they may be useful for patients with metal sensitivity. However, PEEK is a bio-inert material, exhibiting low interaction with proteins and tissues, leading to poor implant-bone integration $^{8,9)}$. Improving osteoblast compatibility is one of the challenges in the utilization of PEEKs as dental materials.

Previously, we reported polyrotaxane (PRX)-based biointerfaces for modulating cellular functions ${ }^{10-14)}$. PRX is a type of supramolecular system in which an axis polymer chain threads into the cavities of multiple cyclic molecules and is capped with bulky molecules at both terminals ${ }^{15-17)}$, and has the potential for molecular

Color figures can be viewed in the online issue, which is available at J-STAGE.

Received Sep 7, 2020: Accepted Oct 12, 2020

doi:10.4012/dmj.2020-332 JOI JST.JSTAGE/dmj/2020-332 mobility, which involves the threading cyclic molecules freely sliding along the chain. For instance, PRXs are formed by a combination of $\alpha$-cyclodextrins ( $\alpha$-CDs) as food additives ${ }^{18)}$ and poly(ethylene glycol)s (PEGs) as biocompatible and water-soluble polymers ${ }^{19}$. When various types of cells were cultured using methylated PRX (MePRX)-coated surfaces with different mobilities, it was observed that the extent of mobility affects morphology and cytoskeletal signaling pathways. When mesenchymal stem cells were induced differentiation on MePRX surfaces, MePRX surfaces with low mobility enhanced the organization of actin filaments to preferentially induce osteoblast differentiation, whereas MePRX surfaces with high mobility suppressed the organization to preferentially induce adipogenic differentiation ${ }^{12}$. Recently, we reported that MePRX surfaces with low mobility show a higher osteoblast compatibility than commercially available tissue culture polystyrene (TCPS) surfaces ${ }^{20)}$, which are the gold standard for cell culture surfaces. When mouse preosteoblasts were cultured on MePRX and TCPS surfaces in an osteoblast differentiation medium, the MePRX surfaces promoted greater proliferation and osteoblast differentiation than the TCPS surfaces.

In the present study, we covalently tethered MePRXs onto PEEK surfaces to improve their osteoblast compatibility. Photoinduced polymerization was performed to covalently tether MePRXs onto PEEK surfaces for long-term use as dental implants in vivo. 
This polymerization method was proposed by Ishihara and coworkers ${ }^{21-23)}$, who demonstrated that the diphenyl ketone units of PEEK act as photo-initiators and that the polymerization of vinyl monomers on PEEK surfaces is initiated by irradiating them with ultraviolet (UV) light. Herein, MePRXs with 4-vinylbenzyl groups ${ }^{24)}$ were covalently tethered onto PEEK surfaces. Subsequently, the proliferation and differentiation of preosteoblasts on the MePRX-tethered and bare PEEK surfaces were investigated.

\section{MATERIALS AND METHODS}

\section{Materials}

MePRX-VBns, composed of methylated $\alpha$-CDs and poly(ethylene glycol) (PEG) chains $\left(M_{\mathrm{n}}=4,700\right)$ capped with 4-vinylbenzyl groups, were prepared according to a procedure described by Arisaka et al. ${ }^{24)}$. Ethanol and dimethyl sulfoxide (DMSO) were purchased from Kanto Chemical (Tokyo, Japan). A PEEK sheet (170 mm×1 $\mathrm{m} \times 0.5 \mathrm{~mm}$ ) was purchased from Sansho Corporation (Tokyo, Japan). PEEK screws were purchased from Nippon Chemical Screw (Tokyo, Japan).

\section{Fabrication of MePRX-tethered PEEK surfaces}

The PEEK substrates were cut into squares $(10 \times 10$ $\mathrm{mm}$ ) and washed with ethanol. Subsequently, they were ultrasonicated for $20 \mathrm{~min}$ and dried at $60^{\circ} \mathrm{C}$ for $18 \mathrm{~h}$. MePRX-VBns $\left(M_{\mathrm{n}}\right.$ of 27,100 , the number of threaded $\alpha$-CDs of 22.2 , the modified number of methyl groups per $\alpha$-CD of 14.1) were dissolved at a concentration of $20 \mathrm{mg} \mathrm{mL} \mathrm{mL}^{-1}$ in DMSO, and $15 \mu \mathrm{L}$ of each solution was spread onto the PEEK surfaces (culture area: $1.0 \mathrm{~cm}^{2}$ ).
After drying at $60^{\circ} \mathrm{C}$ for a further $18 \mathrm{~h}$, the MePRX-VBncoated PEEK surfaces were irradiated with UV light for $90 \mathrm{~min}$ to tether the MePRXs covalently to the PEEK (Fig. 1A). Photostimulation was performed using a UV light-emitting diode (LED; MBRL-CUV7530-2, Moritex, Saitama, Japan) with an emission peak wavelength of $365 \mathrm{~nm}$ and an intensity of $7.03 \mathrm{~mW} \mathrm{~cm}^{-2}$. The surfaces were washed with DMSO and ethanol, and then dried at $60^{\circ} \mathrm{C}$ for $18 \mathrm{~h}$ once more to obtain MePRXVB-tethered PEEK (MePRX-PEEK) surfaces. As a preliminary experiment to demonstrate the concept of surface-functionalizing dental implants using MePRXs, MePRX-tethered PEEK screws were fabricated through a minor modification of the MePRX-PEEK surfaces ${ }^{21}$. For this process, the upper parts of the PEEK screws were masked with poly(tetrafluoroethylene) tape. Subsequently, the surfaces of the screws were dip-coated with MePRX-VBn solution (20 mg $\mathrm{mL}^{-1}$, DMSO). After drying at $60^{\circ} \mathrm{C}$ for $18 \mathrm{~h}$, the screws were UV-irradiated for $90 \mathrm{~min}$. After removing the poly(tetrafluoroethylene) tape, the screws were washed and dried in the same manner as the MePRX-PEEK surfaces.

\section{Characterization of MePRX-tethered PEEK surfaces}

An attenuated total reflectance Fourier transform infrared (ATR-FTIR) spectrometer (Spectrum 100, Perkin Elmer, Waltham, MA, USA) equipped with a Ge crystal ATR unit (VariGATR, Harrick Scientific Products, Pleasantville, NY, USA) was used to detect the characteristic functional groups on the bare PEEK, UV light-irradiated PEEK and MePRX-PEEK surfaces. All the spectra were recorded in the frequency range of $1,000-4,000 \mathrm{~cm}^{-1}$ at a resolution of $4 \mathrm{~cm}^{-1}$ and analyzed
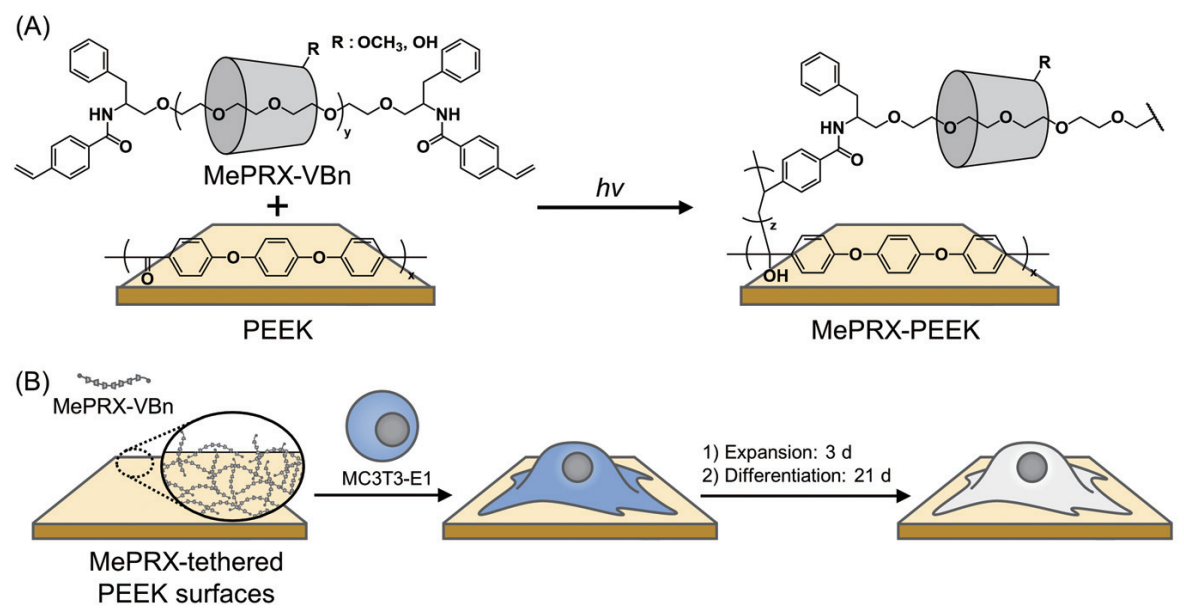

Fig. 1 Schematic illustration of MePRX-VBns tethered onto PEEK surfaces via photo-induced polymerization (A). Inducing the osteoblast differentiation of adhering MC3T3-E1 cells as mouse preosteoblasts on the MePRX-PEEK surfaces (B). PEEK used as the base surface. MePRX-VBn indicates methylated polyrotaxane composed of methylated $\alpha$-cyclodextrins and poly(ethylene glycol) capped with 4-vinylbenzyl groups.

PEEK and MePRX-PEEK surfaces indicate poly(ether ether ketone) and MePRX-tethered PEEK surfaces, respectively. 
using Spectrum Express software (PerkinElmer). Static contact angle analysis was performed in an air atmosphere using a contact angle measurement instrument (DropMaster DM-501, Kyowa Interface Science, Saitama, Japan) for four types of PEEK surfaces: bare, MePRX-VBn-coated, UV light-irradiated, and UV light-irradiated MePRX-VBn-coated PEEK surfaces. All samples were washed with DMSO and ethanol and then dried at $60^{\circ} \mathrm{C}$. A single water droplet $(5 \mu \mathrm{L})$ was placed on each surface and monitored using a charge-coupled device camera at $23^{\circ} \mathrm{C}$. The captured images were analyzed using FAMAS version 5.0.0 software (Kyowa Interface Science).

\section{Proliferation of preosteoblasts using MePRX-tethered PEEK surfaces}

MC3T3-E1 subclone 4, a preosteoblastic cell line established from a mouse calvaria, was obtained from the American Type Culture Collection (Manassas, VA, USA). The MC3T3-E1 cells were expanded on TCPS surfaces (Thermo Fisher Scientific, Waltham, MA, USA) in $\alpha$-minimum essential medium ( $\alpha$-MEM, FUJIFILM Wako) supplemented with $10 \%$ fetal bovine serum (Thermo Fisher Scientific), 1\% penicillin (100 $\mathrm{U} \mathrm{mL}^{-1}$ ), and streptomycin (100 $\mathrm{mg} \mathrm{mL}^{-1}$ ) (FUJIFILM Wako) as a general medium (GM) in a humidified $5 \% \mathrm{CO}_{2}$ atmosphere at $37^{\circ} \mathrm{C}$. MePRX-PEEK and bare PEEK surfaces were set on 24-well culture plates (Thermo Fisher Scientific) and sterilized using UV light irradiation for 20 min on a clean bench. MC3T3-E1 cells were seeded onto each surface at a density of $5.0 \times 10^{3}$ cells $\mathrm{cm}^{-2}$ and expanded using GM at $37^{\circ} \mathrm{C}$ in a humidified atmosphere with $5 \% \mathrm{CO}_{2}$ for 3 days. The nuclear DNA in the adhering cells was stained with Hoechst 33342 (Dojindo, Kumamoto, Japan) (1:500) at intervals of 1 day to count the number of adhering cells. After being stained and washed with phosphate-buffered saline, the cell-adhering surfaces were placed upside down on glass slides to observe their cellular nuclei using a fluorescence microscope (IX71, Olympus, Tokyo, Japan) with cellSens standard software (Olympus). The number of stained nuclei was analyzed from the captured fluorescence images using ImageJ software (NIH, Bethesda, MD, USA). The doubling time of MC3T3-E1 cells on each surface was calculated from the change in the number of adherent cells from $24 \mathrm{~h}$ to $72 \mathrm{~h}$.

\section{Differentiation of preosteoblasts using MePRX-tethered PEEK surfaces}

MC3T3-E1 cells were seeded onto MePRX-PEEK and

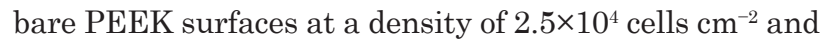
cultured using $\mathrm{GM}$ at $37^{\circ} \mathrm{C}$ in a humidified atmosphere with $5 \% \mathrm{CO}_{2}$ to induce osteogenic differentiation (Fig. 1B). After incubation for 3 days to reach confluence, the medium was replaced with $\alpha$-MEM supplemented with $50 \mu \mathrm{g} \mathrm{mL}^{-1} \mathrm{~L}$-ascorbic acid phosphate magnesium salt, $10 \mathrm{mmol} \mathrm{L}{ }^{-1} \beta$-glycerophosphate disodium salt hydrate, $10 \%$ fetal bovine serum, $100 \mathrm{U} \mathrm{mL}^{-1}$ penicillin, and 100 $\mathrm{mg} \mathrm{mL}^{-1}$ streptomycin as an osteoinductive medium (OIM), followed by incubation for an additional 21 days.
The OIM was refreshed at 3 days intervals. Alizarin red $\mathrm{S}$ staining was performed on the $7 \mathrm{th}, 14 \mathrm{th}$, and $21 \mathrm{st}$ days to evaluate mineralization. The MC3T3-E1 cells on each surface were fixed with $99 \%$ ethanol for 10 min at $23^{\circ} \mathrm{C}$ and stained with $1 \%$ alizarin red $\mathrm{S}$ solution for 10 min at $23^{\circ} \mathrm{C}$. The alizarin red $\mathrm{S}$ solution was removed and rinsed with ultrapure water (Milli-Q). After being photographed, the stain was solubilized with $500 \mu \mathrm{L}$ of DMSO. Subsequently, the solution was aliquoted at $200 \mu \mathrm{L}$ per well in a 96 -well multiplate (Thermo Fisher Scientific) to measure the absorbance at $405 \mathrm{~nm}$ using a Varioskan LUX microplate reader (Thermo Fisher Scientific). The alizarin red $\mathrm{S}$ deposited in adhering cells on each surface was calculated from the concentration of alizarin red $\mathrm{S}$ relative to a standard curve.

\section{Statistical analysis}

Significance was analyzed by one-way analysis of variance (ANOVA) followed by Tukey's post hoc test with a value of $p<0.05$ considered statistically significant. All data are presented as mean \pm standard deviation (SD).

\section{RESULTS}

To covalently tether MePRX-VBns onto PEEK surfaces, the surfaces were coated with MePRX-VBns and then irradiated with UV light for $90 \mathrm{~min}$. After washing and drying the surfaces, the ATR-FTIR spectra of MePRXPEEK, UV light-irradiated PEEK and bare PEEK surfaces were analyzed to confirm the presence of MePRXs on the PEEK surfaces (Fig. 2). The spectrum of the MePRX-PEEK surface showed a peak at 3,251 $\mathrm{cm}^{-1}$, which was ascribed to the typical O-H stretching vibration of methylated $\alpha$-CD. Contact angle measurements with water droplets were performed

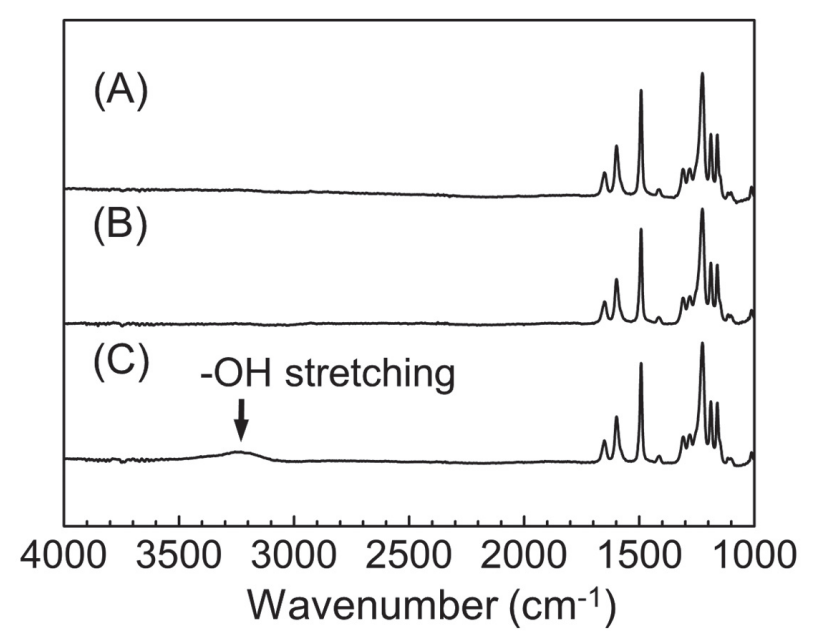

Fig. 2 ATR-FT-IR spectra of (A) bare PEEK, (B) ultraviolet light-irradiated PEEK and (C) MePRXPEEK surfaces.

PEEK and MePRX-PEEK surfaces indicate poly(ether ether ketone) and methylated polyrotaxane-tethered PEEK surfaces, respectively. 


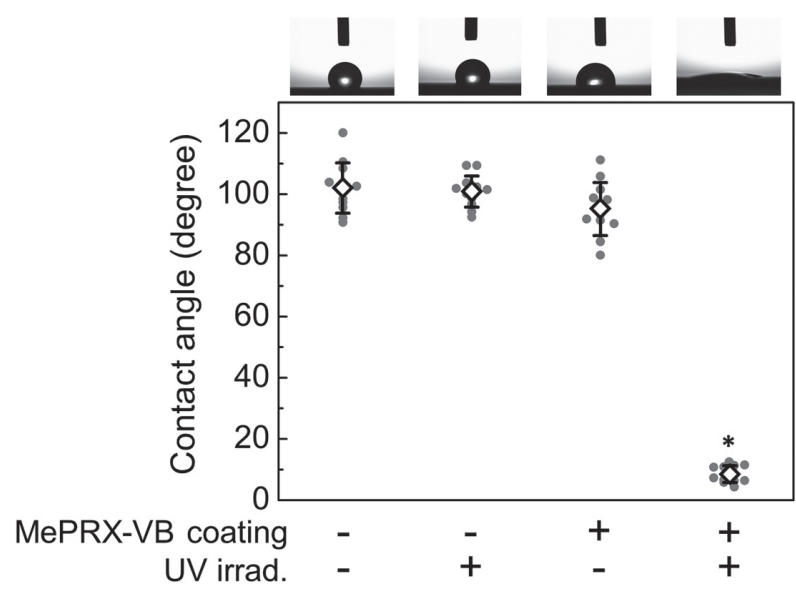

Fig. 3 Contact angle images and values for PEEK surfaces under coating with MePRX-VBns and/or irradiating UV light.

Data of contact angles presented as mean $\pm \mathrm{SD}$, $n=12$. ${ }^{*} p<0.05$ (analysis of variance, ANOVA). MePRX-VBn indicates methylated polyrotaxane composed of methylated $\alpha$-cyclodextrins and poly(ethylene glycol) capped with 4-vinylbenzyl groups. PEEK indicates poly(ether ether ketone).

on PEEK surfaces coated with MePRX-VBns and/or irradiated with UV light to evaluate the wettability of the PEEK surfaces after tethering MePRXs (Fig. 3). The average contact angles for the bare, MePRX-VBn-coated, and UV light-irradiated PEEK surfaces were $102.0^{\circ} \pm 8.2^{\circ}$, $101.0^{\circ} \pm 5.2^{\circ}$, and $95.3^{\circ} \pm 8.7^{\circ}$, respectively. The angle for the UV light-irradiated MePRX-VBns-coated PEEK surfaces was $8.5^{\circ} \pm 2.7^{\circ}$, which is significantly different from the values for the other PEEK surfaces. These results indicate that coating the surfaces with MePRXs or irradiating them with UV light independently had no effect on the wettability of the PEEK surface, whereas the combined treatment increased the wettability of the PEEK surfaces.

The proliferation and differentiation of MC3T3E1 cells were investigated to evaluate the osteoblast compatibility of the MePRX-PEEK and bare PEEK surfaces. When the MC3T3-E1 cells were seeded onto each surface at a density of $5 \times 10^{3}$ cells $\mathrm{cm}^{-2}$, the adhesion ratios of the cells on the MePRX-PEEK and bare PEEK surfaces after 1 day of incubation were $62.6 \%$ and $42.6 \%$, respectively. The doubling times of culturing MC3T3-E1 cells on the MePRX-PEEK and bare PEEK surfaces were $17.6 \mathrm{~h}$ and $20.2 \mathrm{~h}$, respectively (Fig. 4). Alizarin red S staining was performed at intervals of 7 days for 21 days to evaluate the difference between adhering MC3T3-E1 cells on the MePRX-PEEK and bare PEEK surfaces (Fig. 5). The cell-adhering MePRX-PEEK and PEEK surfaces were barely stained with alizarin red $\mathrm{S}$ after cultivation for 7 days in OIM, but became slightly stained after cultivation for 14 days in OIM. After cultivation for 21 days in OIM, the amount of alizarin red $\mathrm{S}$ deposited on cell-adhering MePRX-PEEK surfaces was 2 times higher

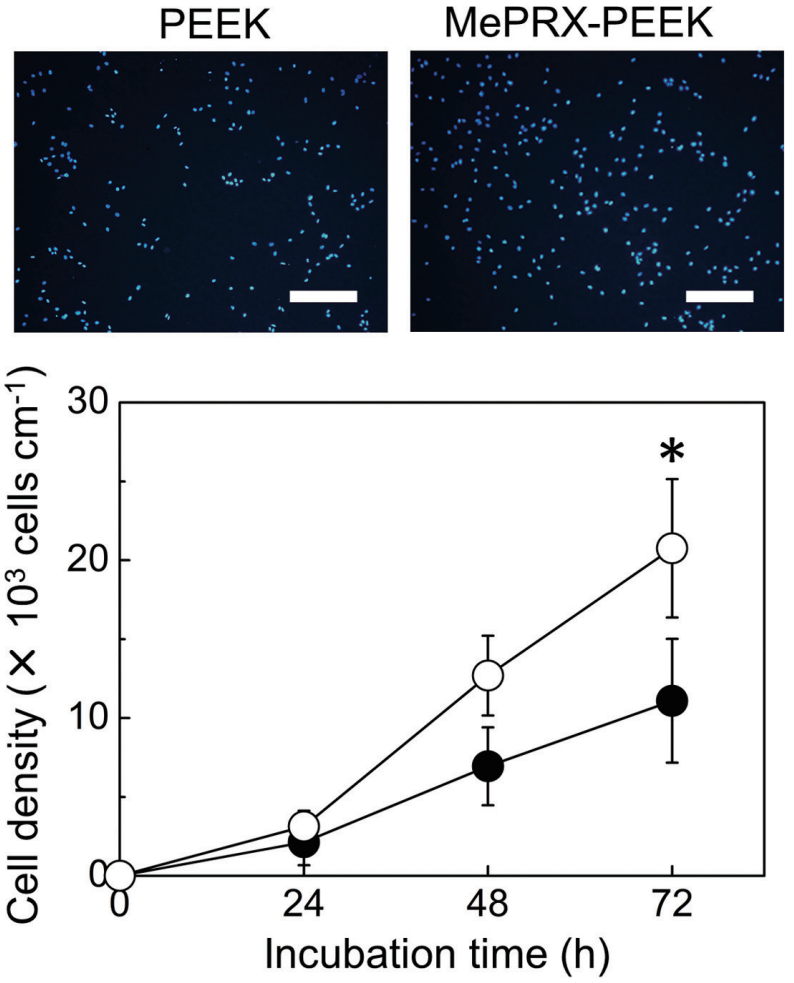

Fig. 4 Fluorescence images of nucleus-stained MC3T3E1 cells on the bare PEEK and MePRX-PEEK surfaces after incubation for 3 days (A). Scale bar: $250 \mu \mathrm{m}$. Time-courses of MC3T3-E1 cells adhering and growing on the bare PEEK (closed circles) and MePRX-PEEK surfaces (open circles) (B). Incubation for 3 days at $37^{\circ} \mathrm{C}$. Cell seeding density was $5.0 \times 10^{3}$ cell s cm$^{-2}$. Data presented as mean $\pm \mathrm{SD}$, $n=6 .{ }^{*} p<0.05$ (analysis of variance, ANOVA). PEEK and MePRX-PEEK surfaces indicate poly(ether ether ketone) and methylated polyrotaxanetethered PEEK surfaces, respectively.

than that on the PEEK surfaces.

\section{DISCUSSION}

Although PEEK is a tough and lightweight engineering plastic with the potential to be used as a medical material for transplantation into hard tissues such as bone, its osteoblast compatibility needs to be improved via surface modification. PEEK has been surface-modified via methods such as spin-coating hydroxyapatite ${ }^{25)}$, gas plasma etching ${ }^{26,27)}$, and sulfonation ${ }^{28)}$. Among them, the photo-initiated polymerization reported by Ishihara and coworkers is a useful method that can polymerize vinyl monomers from PEEK surfaces by irradiating them with UV light without any initiators or catalysts ${ }^{21-23)}$. However, their method could not be directly applied to the surface-tethering of MePRXs onto PEEKs. Although they used $0.5 \mathrm{~mol} \mathrm{~L}^{-1}$ of an aqueous solution containing hydrophilic vinyl monomers ${ }^{21)}$, it was difficult to prepare 


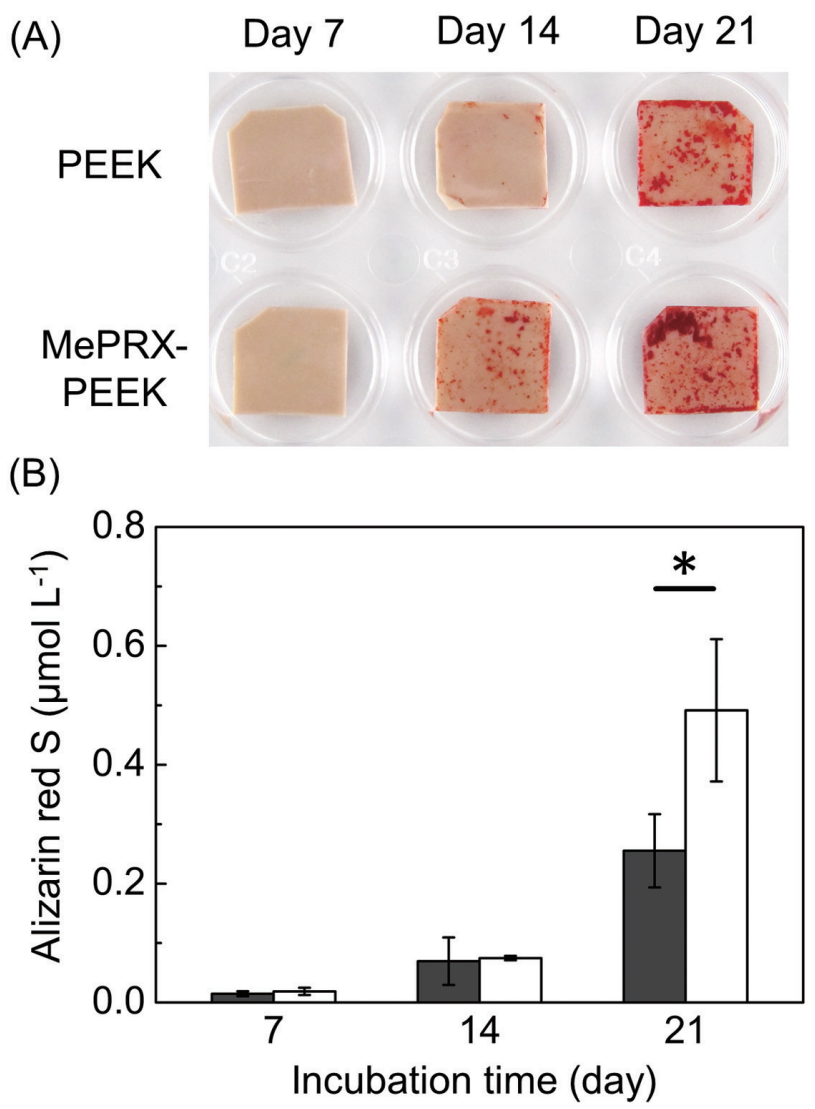

Fig. 5 Alizarin red S staining images for MC3T3-E1 cells as preosteoblasts on the bare PEEK and MePRXPEEK surfaces (A). Concentration of staining with alizarin red $\mathrm{S}$ on each surface after culturing for 7 , 14, and 21 days in OIM (B). Cell seeding density was $2.5 \times 10^{4}$ cells $\mathrm{cm}^{-2}$.

Data presented as mean $\pm \mathrm{SD}, n=3 .{ }^{*} p<0.05$ (analysis of variance, ANOVA). PEEK and MePRX-PEEK surfaces indicate poly(ether ether ketone) and methylated polyrotaxane-tethered PEEK surfaces, respectively.

MePRX-VBn solutions at the same molar concentration, since the MePRX-VBns used as macromonomers have a high molecular weight (ca. 27,100). Therefore, we modified the aforementioned method slightly to perform the surface-tethering of MePRXs. After coating the PEEK surfaces with MePRX-VBns and drying them, the surfaces were irradiated with UV light in an air atmosphere to covalently tether the MePRX-VBns. When the PEEK surfaces were coated with MePRX-VBns and irradiated with UV light using ATR-FTIR instruments, a new peak derived from MePRXs was confirmed in the spectrum. This suggests that MePRXs were tethered to the PEEK surface, although it is difficult to quantify the tethering quality. When the wettability of the PEEK surfaces after irradiation with UV light was analyzed, the surfaces showed the same contact angle as that of the bare PEEK surfaces. It has been reported that
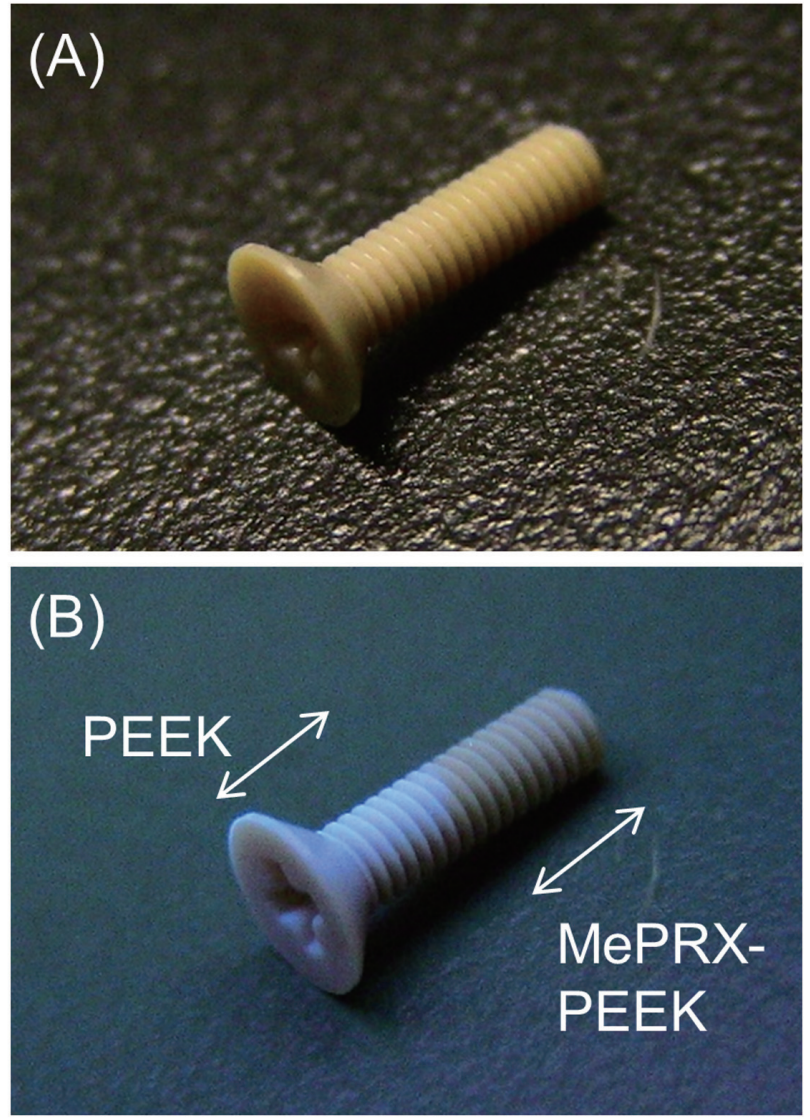

Fig. 6 Photographic images of MePRX-tethered PEEK screws under (A) room light and (B) UV light (360 $\mathrm{nm})$.

Treatment for tethering MePRXs was applied to the lower parts of the PEEK screws. PEEK and MePRX-PEEK indicate poly(ether ether ketone) and methylated polyrotaxanes, respectively.

PEEK surfaces under UV light irradiation become more hydrophilic than bare PEEK surfaces ${ }^{29,30)}$. Although UV light in the UVC region (wavelength $280 \mathrm{~nm}$ or less) has been used for the surface modification of polymer-based materials, it is expected that the UV light of wavelength $365 \mathrm{~nm}$ used in this experiment hardly affected the wettability of the PEEK surfaces. Subsequently, the contact angle for the PEEK surfaces after coating with MePRX-VBns was not significantly different from that of the bare PEEK surfaces. This result suggests that the coated MePRX-VBns were removed from the PEEK surfaces by the DMSO and ethanol wash because of the weak interaction between the MePRX-VBns and the PEEK surfaces. Moreover, the PEEK surfaces subjected to both coating with MePRX-VBns and UV irradiation became significantly hydrophilic, suggesting that the water-soluble MePRX-VBns were tethered to the PEEK surfaces. This is consistent with the results of the ATR-FTIR measurements. Furthermore, the MePRXPEEK surfaces were observed to be superhydrophilic through contact angle measurements. The wettability 
of materials is one of the key properties controlling their interactions with proteins and cells $\mathrm{s}^{31-33)}$. It has been reported that superhydrophilic PEEK surfaces fabricated via the combination of sulfonation and UV irradiation show a higher fibronectin adsorption and bone-to-implant contact ratio than as-polished PEEK surfaces $^{34)}$. In the present study, seeding with MC3T3E1 cells onto MePRX-PEEK surfaces resulted in a higher proliferation and osteoblast differentiation than that of the bare PEEK surfaces, which is consistent with the above report. Previously, we have succeeded in promoting osteoblast differentiation of human mesenchymal stem cells by modulating the mobility of threading $\alpha$-CDs ${ }^{14)}$. In addition, we have reported that sulfonated polyrotaxanes form polyelectrolyte complexes with bone morphogenetic protein-2 (BMP-2), enhancing

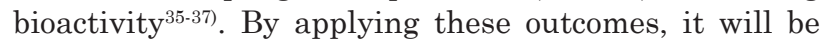
possible to further improve the osteoblast compatibility of PEEK surfaces.

Finally, PEEK screws were surface-modified as a preliminary demonstration for their application to dental implants with complicated shapes. When tethering treatment was applied to the lower half of the PEEK screws, the autofluorescence of the PEEK screws in the lower half disappeared (Fig. 6). Although PEEK is known to have a strong fluorescence in the wavelength range of $350-550 \mathrm{~nm}^{38)}$, the disappearance of autofluorescence indicates that MePRXs may have been covalently tethered at specific areas on the surfaces of the screws.

\section{CONCLUSION}

We demonstrated the surface-tethering of MePRXs with 4-vinylbenzyl groups onto PEEK surfaces via a minor modification of photo-induced polymerization using PEEKs as the photo-initiators. MePRXs tethered onto PEEK surfaces promoted the proliferation and osteoblast differentiation of MC3T3-E1 cells and contributed to the improvement of osteoblast compatibility on the PEEK surfaces.

\section{REFERENCES}

1) Searle OB, Pfeiffer RH. Victrex ${ }^{\mathbb{R}}$ poly (ethersulfone) (PES) and Victrex ${ }^{\circledR}$ poly (etheretherketone) (PEEK). Polym Eng Sci 1985; 25: 474-476.

2) Nguyen HX, Ishida H. Poly (aryl-ether-ether-ketone) and its advanced composites: A review. Polym Compos 1987; 8: 5773.

3) Segal D. Materials for the 21st Century. Oxford: Oxford University Press; 2017.

4) Schwitalla AD, Spintig T, Kallage I, Müller WD. Flexural behavior of PEEK materials for dental application. Dent Mater J 2015; 31: 1377-1384.

5) Ma R, Tang T. Current strategies to improve the bioactivity of PEEK. Int J Mol Sci 2014; 15: 5426-5445.

6) Bathala L, Majeti V, Rachuri N, Singh N, Gedela S. The Role of Polyether Ether Ketone (Peek) in Dentistry -A Review. J Med Life 2019; 12: 5-9.

7) Najeeb S, Zafar MS, Khurshid Z, Siddiqui F. Applications of polyetheretherketone (PEEK) in oral implantology and prosthodontics. J Prosthodont Res 2016; 60: 12-19.

8) Liu X, Han F, Zhao P, Lin C, Wen X, Ye X. Layer-bylayer self-assembled multilayers on PEEK implants improve osseointegration in an osteoporosis rabbit model. Nanomedicine 2017; 13: 1423-1433.

9) Olivares-Navarrete R, Gittens RA, Schneider JM, Hyzy SL, Haithcock DA, Ullrich PF, et al. Osteoblasts exhibit a more differentiated phenotype and increased bone morphogenetic protein production on titanium alloy substrates than on polyether-ether-ketone. Spine J 2012; 12: 265-272.

10) Seo JH, Kakinoki S, Inoue Y, Yamaoka T, Ishihara K, Yui N. Designing dynamic surfaces for regulation of biological responses. Soft Matter 2012; 8: 5477-5485.

11) Seo JH, Yui N. The effect of molecular mobility of supramolecular polymer surfaces on fibroblast adhesion. Biomaterials 2013; 34: 55-63.

12) Seo JH, Kakinoki S, Yamaoka T, Yui N. Directing stem cell differentiation by changing the molecular mobility of supramolecular surfaces. Adv Healthcare Mater 2015; 4: 215222.

13) Seo JH, Hirata M, Kakinoki S, Yamaoka T, Yui N. Dynamic polyrotaxane-coated surface for effective differentiation of mouse induced pluripotent stem cells into cardiomyocytes. RSC Adv 2016; 6: 35668-35676.

14) Seo JH, Kakinoki S, Inoue $Y$, Nam K, Yamaoka T, Ishihara K, et al. The significance of hydrated surface molecular mobility in the control of the morphology of adhering fibroblasts. Biomaterials 2013; 34: 3206-3214.

15) Harada A, Li J, Kamachi M. The molecular necklace: a rotaxane containing many threaded $\alpha$-cyclodextrins. Nature 1992; 356: 325-327.

16) Wenz G, Han BH, Müller A. Cyclodextrin rotaxanes and polyrotaxanes. Chem Rev 2006; 106: 782-817.

17) Harada A, Hashidzume A, Yamaguchi H, Takashima Y. Polymeric rotaxanes. Chem Rev 2009; 109: 5974-6023.

18) Astray G, Gonzalez-Barreiro C, Mejuto JC, Rial-Otero R, Simal-Gandara J. A review on the use of cyclodextrins in foods. Food Hydrocoll 2009; 23: 1631-1640.

19) Knop K, Hoogenboom R, Fischer D, Schubert US. Poly (ethylene glycol) in drug delivery: pros and cons as well as potential alternatives. Angew Chem Int 2010; 49: 6288-6308.

20) Masuda H, Arisaka Y, Sekiya-Aoyama R, Yoda T, Yui N. Biological effects of polyrotaxane surfaces on cellular responses of fibroblast, preosteoblast and preadipocyte cell lines. Polymers 2020; 12: 924.

21) Kyomoto M, Ishihara K. Self-initiated surface graft polymerization of 2-methacryloyloxyethyl phosphorylcholine on poly (ether ether ketone) by photoirradiation. ACS Appl Mater Inter 2009; 1: 537-542.

22) Kyomoto M, Moro T, Yamane S, Watanabe K, Takatori $\mathrm{Y}$, Tanaka S, et al. Smart PEEK modified by self-initiated surface graft polymerization for orthopedic bearings. Reconstr Rev 2014: 4(3).

23) Kyomoto M, Moro T, Takatori Y, Kawaguchi H, Nakamura $\mathrm{K}$, Ishihara K. Self-initiated surface grafting with poly (2-methacryloyloxyethyl phosphorylcholine) on poly (etherether-ketone). Biomaterials 2010; 31: 1017-1024.

24) Arisaka Y, Tonegawa A, Tamura A, Yui N. Terminally cross-linking polyrotaxane hydrogels applicable for cellular microenvironments. J Appl Polym Sci 2020; 49706.

25) Barkarmo S, Wennerberg A, Hoffman M, Kjellin P, Breding K, Handa P, et al. Nano-hydroxyapatite-coated PEEK implants: A pilot study in rabbit bone. J Biomed Mater Res A 2013; 101: 465-471.

26) Poulsson AH, Eglin D, Zeiter S, Camenisch K, Sprecher C, Agarwal Y, et al. Osseointegration of machined, injection moulded and oxygen plasma modified PEEK implants in a sheep model. Biomaterials 2014; 35: 3717-3728.

27) Waser-Althaus J, Salamon A, Waser M, Padeste C, Kreutzer 
$\mathrm{M}$, Pieles U, et al. Differentiation of human mesenchymal stem cells on plasma-treated polyetheretherketone. J Mater Sci Mater Med 2014; 25: 515-525.

28) Huang RYM, Shao P, Burns CM, Feng X. Sulfonation of poly (ether ether ketone) (PEEK): kinetic study and characterization. J Appl Polym Sci 2001; 82: 2651-2660.

29) Shi H, Sinke J, Benedictus R. Surface modification of PEEK by UV irradiation for direct co-curing with carbon fibre reinforced epoxy prepregs. Int J Adhes Adhes 2017; 73: 5157.

30) Laurens P, Bouali MO, Meducin F, Sadras B. Characterization of modifications of polymer surfaces after excimer laser treatments below the ablation threshold. Appl Surf Sci 2000; 154: 211-216.

31) Wei Q, Becherer T, Angioletti-Uberti S, Dzubiella J, Wischke C, Neffe AT, et al. Protein interactions with polymer coatings and biomaterials. Angew Chem Int 2014; 53: 8004-8031.

32) Vogler EA. Structure and reactivity of water at biomaterial surfaces. Adv Colloid Interface Sci 1998; 74: 69-117.

33) Arima Y, Iwata $\mathrm{H}$. Effect of wettability and surface functional groups on protein adsorption and cell adhesion using welldefined mixed self-assembled monolayers. Biomaterials 2007; 28: 3074-3082.
34) Kuroda K, Igarashi K, Kanetaka H, Okido M. Surface modification of PEEK and its osteoconductivity and antiinflammatory properties. J Biomater Nanobiotechnol 2018; 9: 233-243.

35) Terauchi M, Ikeda G, Nishida K, Tamura A, Yamaguchi S, Harada K, et al. Supramolecular polyelectrolyte complexes of bone morphogenetic protein-2 with sulfonated polyrotaxanes to induce enhanced osteogenic differentiation. Macromol Biosci 2015; 15: 953-964.

36) Terauchi M, Inada T, Kanemaru T, Ikeda G, Tonegawa A, Nishida K, et al. Potentiating bioactivity of BMP-2 by polyelectrolyte complexation with sulfonated polyrotaxanes to induce rapid bone regeneration in a mouse calvarial defect. J Biomed Mater Res A 20178; 105: 1355-1363.

37) Arisaka Y, Yui N. Tethered bone morphogenetic protein-2 onto sulfonated-polyrotaxane based surfaces promotes osteogenic differentiation of MC3T3-E1 cells. J Biomater Sci Polym Ed 2017; 28: 974-985.

38) Novotna Z, Reznickova A, Rimpelova S, Vesely M, Kolska Z, Svorcik V. Tailoring of PEEK bioactivity for improved cell interaction: plasma treatment in action. RSC Adv 2015; 5: 41428-41436. 